The Free Internet Journal

for Organic Chemistry
Paper

Arkivoc 2017, part iv, 194-209

\title{
Synthesis of cis-enamide macrocycles via ring-closing metathesis
}

\section{Moisés Ramírez, Adrián Ochoa-Terán, Ratnasamy Somanathan, and Gerardo Aguirre*}

Centro de Graduados e Investigación en Química. Instituto Tecnológico de Tijuana. Blvd. Alberto Limón Padilla S/N, Mesa de Otay, 22510 Tijuana, B.C. México

E-mail: gaguirre@tectijuana.mx

Received 01-12-2017

Accepted 02-25-2017

Published on line 05-21-2017

\section{Abstract}

Herein we report the synthesis of 13-, 14- and 15-membered cyclic lactams, using Grubbs' RCM method in 25, 52 and $46 \%$ yields respectively. The cis-enamide functional group was successfully introduced into these cyclic lactams by syn sulfoxide elimination The synthetic cyclic lactams resemble natural cyclic peptides. Our synthetic methodology provides a simple route to making medium-sized cyclic lactams that could be used as models to mimic the $\beta$-turn in natural proteins, an important marker in understanding their biological activity.<smiles>[R]c1cc([R])c(C=O)cc1O</smiles><smiles>C=CCCC</smiles><smiles>[R]c1cc([R])c(C(CNC(=O)NC=C)Sc2ccccc2)cc1OCC=C</smiles><smiles>C=CCCC</smiles><smiles>[Y]/C=C\COc1cc(/C=C\NC(=O)O)c([R2])cc1[R]</smiles>

$$
\begin{aligned}
& \mathrm{R}^{1}=\mathrm{H}, \mathrm{Br} \\
& \mathrm{R}^{2}=\mathrm{H}, \mathrm{Br}=2-4
\end{aligned}
$$

Keywords: cyclopeptide alkaloids, ring closing metathesis, sulfoxide elimination 


\section{Introduction}

Over the past five decades, about 500 cyclopeptides have been isolated from over 120 species. A large number of 13-, 14- and 15-membered cyclopeptides have been isolated from plants belonging to the Rhamnaceae, Sterculiaceae, Pandaceae, Rubiaceae, Urticaceae, Hymenocardiaceae, and Celastraceae families. ${ }^{1-6}$ We are interested in the chemistry of the cyclic lactams with an enamide (styrylamide) functionality in their cyclic structure, e.g. zizyphine $A,{ }^{7}$ or a secondary alcohol group, which probably is the precursor to the styrylamide through dehydration, as in pandamine (Figure 1). ${ }^{8}$ Plant cyclopeptides have shown various bioactivities, such as antitumor, antibacterial, antifungal, antiHIV, antimalarial, antiplasmodial, insecticidal, antiplatelet, cyclooxygenase and tyrosine inhibiting activities. ${ }^{9-13}$

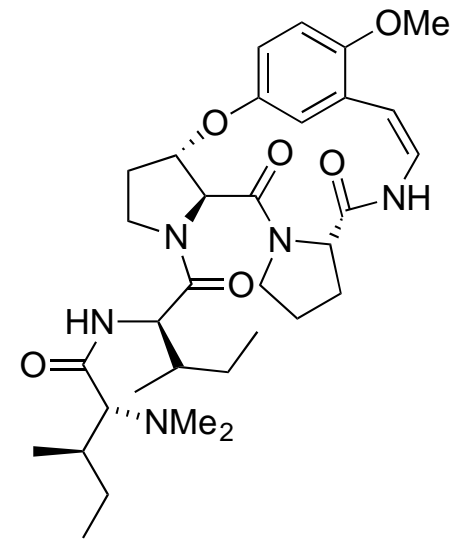

zizyphine $\mathrm{A}$

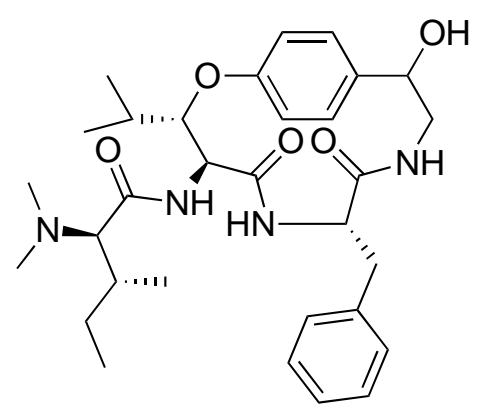

pandamine

Figure 1. Same examples of cyclic peptides.

Our interest in the chemistry of enamides (styrylamides) arose from our ongoing program of study of the synthesis and biological activity of acyclic enamides, like tuberine and amathamide A (Figure 2). ${ }^{14-18}$ Many of the acyclic enamides isolated from marine sources as ascidians, ${ }^{19-23}$ microorganisms, ${ }^{24-26}$ fungal and higher plants ${ }^{27-31}$ exhibit a variety of pharmacological activities. ${ }^{25,26,32,33}$ Especially the macrolides salicylihalamides $A$ and $B,{ }^{34,35}$ apicularen $A$ and $B^{36}$ and lobatamide $A-F^{37,38}$ isolated from marine sponge Haliclona sp., Chondromyces sp. (myxobacteria) and tunicates, respectively. Salicylihalamides A, B and lobatamides are highly potent cytotoxic macrolides, incorporating salicylic acid, a 12-membered lactone ring and an enamide side-chain (trans A, cis B) (Figure 2). 
<smiles>COc1ccc(/C=C/NC=O)cc1</smiles>

tuberine<smiles>COc1cc(/C=C/NC(=O)C2CCCN2C)c(Br)cc1Br</smiles>

amathamide $\mathrm{A}$<smiles></smiles>

salicylihalamide A: 17E salicylihalamide B: $17 Z$<smiles>CC/C=C\C=C/C(=O)N/C=C/C[C@H]1C[C@H]2C[C@@H](O)C[C@H](Cc3cccc(O)c3C(=O)O2)O1</smiles>

apicularen $\mathrm{A}$<smiles>CO/N=C/C=C\C(=O)N/C=C/C[C@H](CC(=O)OC(C)/C=C/[C@H](O)/C(C)=C\Cc1cccc(O)c1C(=O)O)OC</smiles>

lobatamide A

Figure 2. Some examples of biologically active acyclic enamides.

Salicylihalamide A and lobatamide A display potent cytotoxicity in the $\mathrm{NCl} 60$-cell line human tumor assay, and it is suggested that these compounds may act by a novel mechanism of action. ${ }^{39}$ Recently, it has been reported that salicylate enamide macrolides selectively inhibit the Vo sector of the V-ATPase through $N$ acyliminium ion generated from $\mathrm{N}$-acyl enamines under acidic conditions. ${ }^{39}$ Figure 4 shows how the acyliminium is captured by a nucleophilic amino acid side-chain, and subsequently undergoes irreversible hydrolysis. A study on the structure-activity relationships (SAR) of (-)-salicylihalamide A reported by Barbander and co-workers, revealed that the characteristic $N$-acyl enamine functionality is essential for the biological activity. ${ }^{40,41,42}$ This mechanistic route via the enamide ATPase protein interaction, its biological implications, and its stereochemistry attracted our attention and prompted us to develop a simple and efficient synthetic route to the acyclic enamide tuberine and its analogs, in order to study in detail their biological properties. ${ }^{14-18}$ 


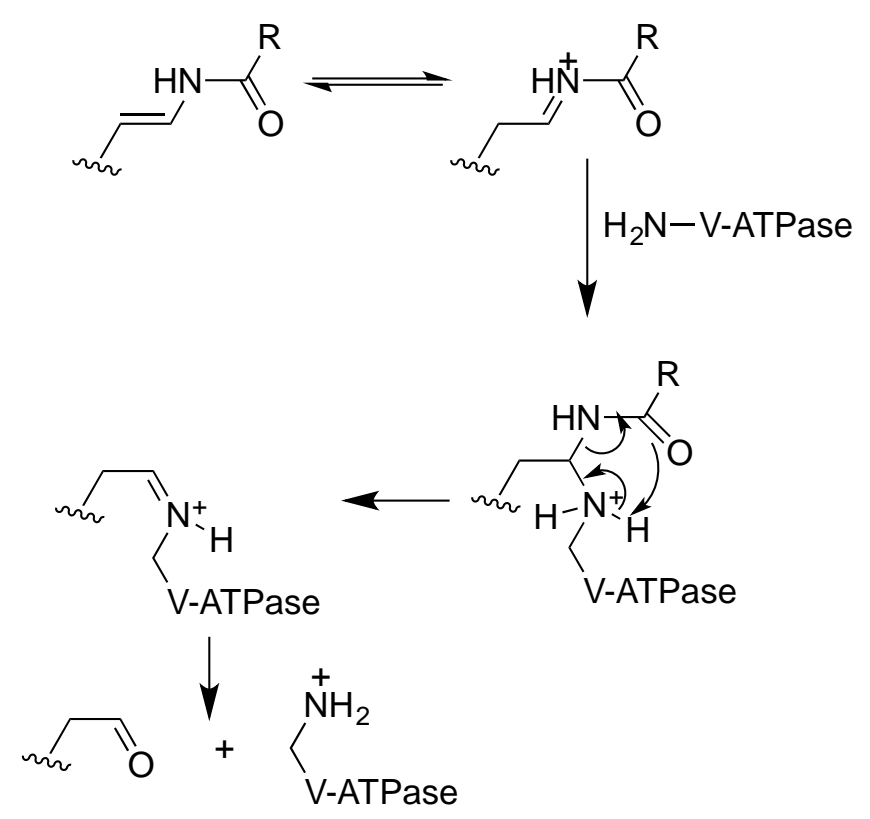

Figure 3. Mechanism of action.<smiles>COc1ccc(C[C@H](NC(=O)/C=C/C[C@H](OC(=O)[C@H](CC(C)C)OC(=O)C(C)(C)CNC(=O)[C@H]2OC2c2ccccc2)C(C)C)C(=O)O)cc1Cl</smiles>

cryptophycim 52

(anticancer)

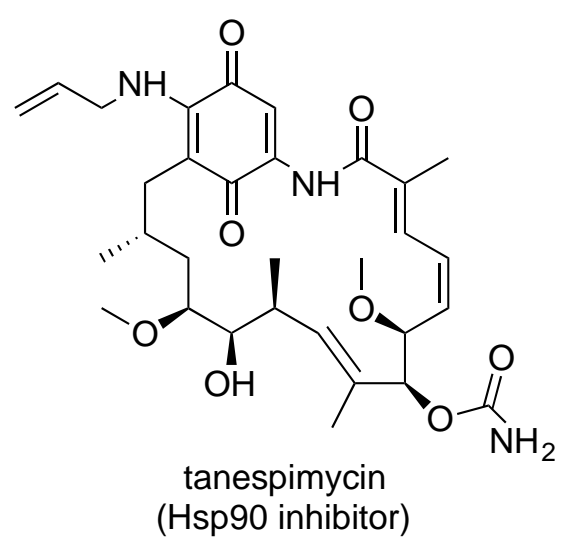

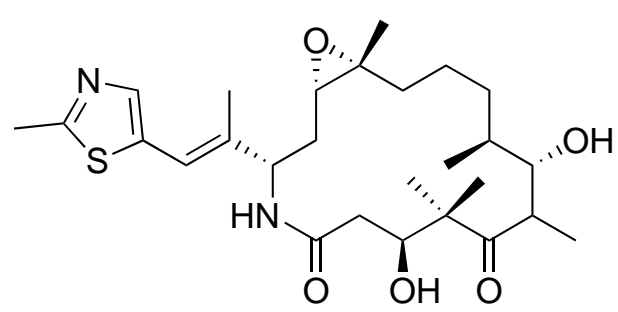

ixabepilone (anticancer)

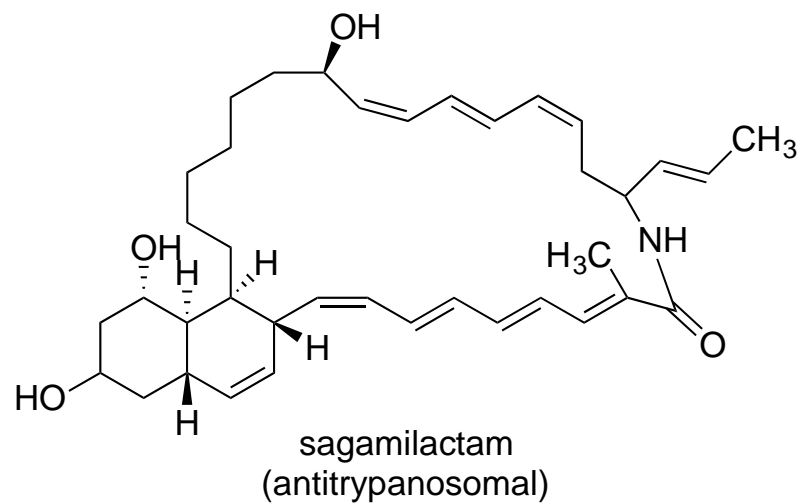

Figure 4. Biologically active macrocyclic lactams and enamides.

Biologically active macrocylic lactams and enamides have been reported, isolated from natural sources. The cryptophycins are a family of highly cytotoxic, cyclic depsipeptides and are among the the most potent agents, and their binding to tubulin is very strong and poorly reversible. Many cryptophycins have been 
isolated fron Cyanobacterium nostoc sp. ${ }^{43}$ Cryptophycin 52 (Figure 4) was produced by total chemical synthesis and it destroys microtubules in vascular smooth muscle cells. Ixabepilone is a semisynthetic epothilone B analogue, which acts like Paclitaxel as a microtubule stabilizing agent. It is used in the treatment of aggresive metastatic or locally advanced breast cancer. ${ }^{44}$ Tanespimycin is a derivative of the antibiotic geldanamycin that is being studied in the treatment of cancer, specifically in young patients with certain types of leukemia or solid tumors, especially kidney tumors. It works by inhibiting Hsp90, which is expressed in those tumors, and it belongs to the family of drugs called antitumor antibiotics. ${ }^{45}$ Finally, sagamilactam is a polyunsaturated and polyoxygenated macrocyclic lactam containing diene, triene and tetraene conjugated olefins and a decalin moiety which has antitrypanosomal activity. ${ }^{46}$

(a)

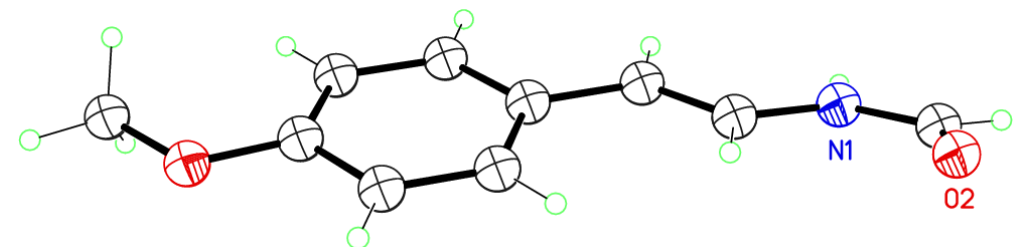

(b)

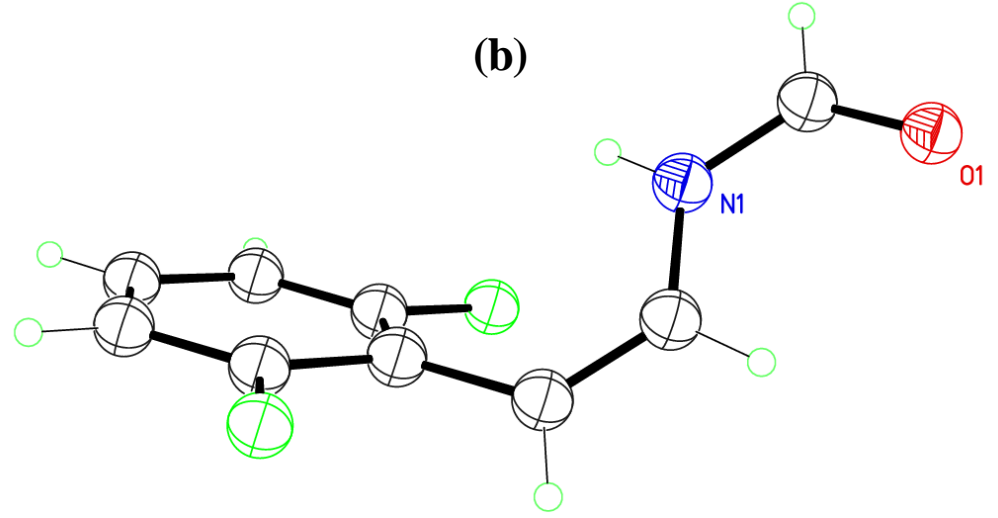

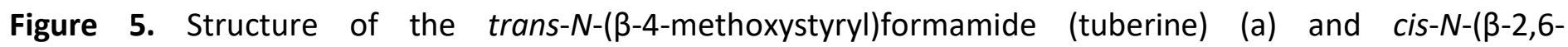
difluorostyryl)formamide (b).

In our synthesis of tuberine and other simple acyclic enamides, the method we used involved thermal synelimination of $\beta$-sulfoxide derived from an amide to generate the enamide functional group. ${ }^{15-18}$ This method was successfully employed in the synthesis of some naturally occurring $N$-acylenamides. ${ }^{16}$ In most cases when the $N$-acyl is a formyl group, the $Z-N$-alkenylformamides were the major product. The naturally occurring tuberine also exhibited a similar Z:E ratio. In addition, ${ }^{1} \mathrm{H}$ NMR signals for the two stereoisomers due to the conformers arising from restricted amide bond rotation were encountered, which led us to calculate a rotational barrier of $19 \mathrm{kcal} / \mathrm{mol}$ using 2D EXY NMR. Since many of the naturally occurring enamides also have a cis-enamide, it was of interest to study in depth the physical properties of cis-enamides. The cis- $N-(\beta-2,6-$ difluorostyryl)formamide was selected as a model. The NMR studies revealed a rotational barrier for the cisenamide with the same magnitude as the trans-enamide. However, the X-ray structure of the trans-trans- $N$ ( $\beta$-4-methoxystyryl)formamide (tuberine) showed that the benzene $\pi$-plane was coplanar to the $\pi$-enamide bond (Figure $5 a)$, while in the cis-enamide $N$-( $\beta$-2,6-difluorostyryl)formamide (Figure $5 b)$ the styrylamide $\pi$ - 
plane of the double bond was at 48 degrees with respect to the benzene ring $\pi$-plane. These structures agree well with computational models. A similar conformational behavior in the molecular modelling study of sanjonine G1 was observed by Zhu et al., where the $\sigma$ bond in the macrocyle lies out of the plane defined by the aromatic ring.

This subtle difference in the orientation of the double bond in the trans and cis enamide function and the biological significance prompted us to synthesize and study the biological properties of some model 13-, 14and 15-membered cyclic enamides and explore the relationship between stereochemistry and biological properties. Our goal in this project was to attempt and introduce the cis enamide functionality into a cyclic 13, 14- and 15-sized lactams, using a syn-sulfoxide elimination methodology, and to study these compounds as models for the natural cyclopeptides.

\section{Results and Discussion}

Our synthetic strategy for the high membered macrocycles is shown in Scheme 1 . It started with the alkylation of an appropriately substituted hydroxybenzaldehyde $\mathbf{1}$ with allyl bromide under basic conditions. The allyloxybenzaldehydes $\mathbf{2}$ were subjected to an addition of nitromethane, followed by a subsequent Michael addition of thiophenol to nitrostyrenes $\mathbf{3}$ to give nitro sulfides $\mathbf{4}$. The nitro group was reduced to amino giving compounds $\mathbf{5}$, and these were then derivatized with a suitable $n$-alkenyl carboxylic acid to give the amides 6-8 with a diene functionality, which then were ring closed by the RCM method using a GrubbsHoveyda first generation catalyst to give the macrocyclic compounds 9-11 in very good yields. The sulfide group was oxidized to sulfoxide and the sulfoxides subjected to thermal elimination to give the cis-enamides 12-14 in good yields. This methodology is straightforward compared to the selenocyanate oxidative elimination reported independently by Schmidt and Joullie in the synthesis of the cyclopeptide alkaloids franguline $A$ and nummularine $F$, respectively. ${ }^{47}$ Our initial goal was to find a suitable methodology to construct the required ring system, followed by a syn elimination of the sulfoxide to generate the enamide functional group. Among the various methods known for making cyclopeptides, ethers and esters, catalytic ring-closing olefin metathesis is by far the simplest and most useful tool for making medium-sized ring compounds. ${ }^{48-56}$ 
<smiles>[R7]c1cc([R7])c(C=O)cc1O</smiles><smiles>C[10BH]C</smiles>
$R^{1} R^{2}$

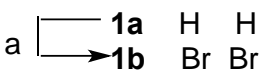<smiles>[R]c1cc([R])c(C(CNC(=O)NCC)Sc2ccccc2)cc1OCC=C</smiles>

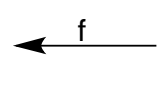

$R^{1} R^{2}$

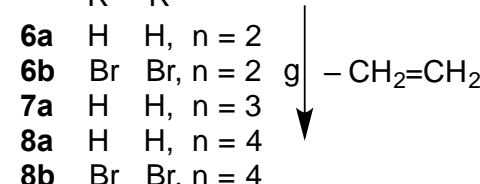<smiles>[R]c1cc([R])c(C(CNC(=O)c2ccccc2)Sc2ccccc2)cc1OC/C=C\C(=O)NC</smiles>

$R^{1} R^{2}$

9a $\mathrm{H} \quad \mathrm{H}, \mathrm{n}=2$

9b $\mathrm{Br} \quad \mathrm{Br}, \mathrm{n}=2$

10a $\mathrm{H} \quad \mathrm{H}, \mathrm{n}=3$

11a $\mathrm{H} \quad \mathrm{H}, \mathrm{n}=4$

11b $\mathrm{Br} \quad \mathrm{Br}, \mathrm{n}=4$<smiles>[R7]c1cc([R])c(OCC=C)cc1C=O</smiles>

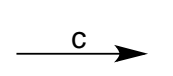<smiles>[R]c1cc([R2])c(OCC=C)cc1/C=C/[N+](=O)[O-]</smiles>

2a $\mathrm{H}$

2b $\mathrm{Br} \mathrm{Br}$

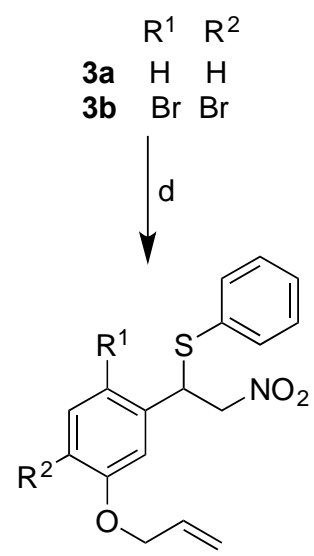

$\begin{array}{lll}\text { 4a } & \mathrm{R}^{1} & \mathrm{R}^{2} \\ \text { 4b } & \mathrm{B} & \mathrm{H} \\ \mathbf{4 r} & \mathrm{Br}\end{array}$

Scheme 1. Synthesis of 13, 14 and 15-member lactams via RCM. Reagents and conditions: (a) $\mathrm{CHCl}_{3}, \mathrm{Br}_{2}$; (b) $\mathrm{C}_{3} \mathrm{H}_{5} \mathrm{Br}, \mathrm{DMF}, \mathrm{K}_{2} \mathrm{CO}_{3}$; (c) $\mathrm{CH}_{3} \mathrm{NO}_{2}, \mathrm{AcOH}, \mathrm{AcONH}_{4}$; (d) $\mathrm{PhSH}, \mathrm{N}$-Isopropyl-cyclohexylamine, $\mathrm{CH}_{2} \mathrm{Cl}_{2}$; (e) $\mathrm{Zn}, \mathrm{HCl}$, $\mathrm{AcOH}$ or $\mathrm{Sml}_{2}, \mathrm{MeOH}$; (f) DCC, DMAP, $n$-alkenyl carboxylic acid or $n$-alkenyl acyl chloride; (g) Grubbs catalyst, $\mathrm{DCM}$; (h) i. $\mathrm{NaIO}_{4}$; ii toluene, $\mathrm{K}_{2} \mathrm{CO}_{3}$.

\section{Conclusions}

We have synthesized 13-, 14- and 15-membered cis-enamide macrocycles using Grubbs RCM method in 25, 52 and $46 \%$ overall yields, respectively. The cis-enamide functional group was successfully introduced into these cyclic lactams by a syn sulfoxide elimination. This synthetic methodology provides a simple route to making medium-sized cyclic lactams. These medium-sized cyclic lactams could be used as models to mimic the $\beta$-turn in natural proteins, an important marker in understanding their biological activity. ${ }^{57-60}$ 


\section{Experimental Section}

General. All reagents were purchased in the highest quality available and were used without further purification. ${ }^{1} \mathrm{H}$ and ${ }^{13} \mathrm{C}$ NMR spectra were recorded using a Varian $500 \mathrm{MHz}^{2}$ pectrometer in $\mathrm{CDCl}_{3}$ with $\mathrm{TMS}$ as internal standard and $200 \mathrm{MHz}$. The chemical shifts are expresed in ppm and the coupling constants $(J)$ in Hertz. Electronic impact mass spectra were obtained by direct insertion in Agilent 5975C mass spectrometer. Infrared spectra were recorded on a Spectrum FT-IR 1600 spectrophotometer. Melting points were determined on a Fisher-Johns melting point apparatus and were corrected.

2,4-Dibromo-5-hydroxybenzaldehyde (1b). To a solution of 3-hydroxybenzaldehyde 1a $(40.9 \mathrm{mmol}, 5.0 \mathrm{~g})$ in $\mathrm{CHCl}_{3}(100 \mathrm{~mL})$ was added bromine $(81.7 \mathrm{mmol}, 4.2 \mathrm{~mL})$. The resulting solution was stirred at $\mathrm{rt}$ for $3 \mathrm{~d}$. The excess bromine was removed with a saturated solution of sodium thiosulfate $(20 \mathrm{~mL})$, the organic phase was washed with water and dried over anhydrous $\mathrm{Na}_{2} \mathrm{SO}_{4}$. Removal of solvent gave a solid which was recrystallized

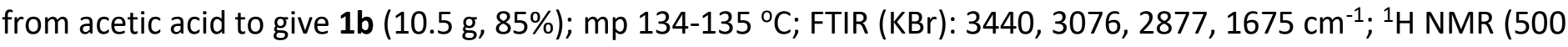
$\mathrm{MHz}_{\mathrm{CDCl}}$ ): $\delta 10.23$ (s, 1H), 7.80 (s, 1H), 7.54 (s, 1H), 6.33 (brs, $\left.\left.1 \mathrm{H}\right) ;{ }^{13} \mathrm{C} \mathrm{NMR} \mathrm{(125} \mathrm{MHz,} \mathrm{CDCl}\right): \delta 190.9$, $152.5,136.5,133.8,117.8,116.8,116.2 ; \mathrm{MS}(\mathrm{EI}) \mathrm{m} / \mathrm{z}: 280\left(\mathrm{M}^{+}, 15\right), 63$ (100).

3-Allyloxybenzaldehyde (2a). To a solution of 3-hydroxybenzaldehyde 1a (81.9 mmol, $10.0 \mathrm{~g})$ and $\mathrm{K}_{2} \mathrm{CO}_{3}(1.5$ $\mathrm{g})$ in DMF $(100.0 \mathrm{~mL})$ was added allyl bromide $(7.8 \mathrm{~mL})$ and the mixture was stirred for $3 \mathrm{~h}$ at $\mathrm{rt}$. The reaction mixture was quenched with water $(50 \mathrm{~mL})$ and the organic phase was extracted into $\mathrm{Et}_{2} \mathrm{O}(3 \times 100 \mathrm{~mL})$, dried over $\mathrm{Na}_{2} \mathrm{SO}_{4}$ and the solvent removed at reduced pressure to give a yellow liquid $2 \mathrm{a}(11.0 \mathrm{~g}, 83 \%): \mathrm{FTIR}(\mathrm{KBr})$ 3071, 2818, $1697 \mathrm{~cm}^{-1} ;{ }^{1} \mathrm{H}$ NMR $(200 \mathrm{MHz}): \delta 9.91(\mathrm{~s}, 1 \mathrm{H}), 7.41(\mathrm{~m}, 3 \mathrm{H}), 7.17(\mathrm{~m}, 1 \mathrm{H}), 6.04(\mathrm{~m}, 1 \mathrm{H}), 5.41(\mathrm{dd}, J$ 16.0, $6.0 \mathrm{~Hz}, 1 \mathrm{H}$ ), 5.30 (dd, J 10.0, $6.0 \mathrm{~Hz}, 1 \mathrm{H}), 4.58$ (d, J $5.6 \mathrm{~Hz}, 2 \mathrm{H}) ;{ }^{13} \mathrm{C} \mathrm{NMR}\left(50 \mathrm{MHz}, \mathrm{CDCl}_{3}\right): \delta$ 191.7, 159.1, 137.7, 132.8, 130.1, 123.6, 122.1, 118.0, 113.1, 68.9; MS(EI) m/z: 162 (M+, 100), 147 (47), 121 (94), 65 (81).

5-Allyloxy-2,4-dibromobenzaldehyde (2b). Compound 2b was synthesized from compound $\mathbf{1 b}$ (17.85 mmol, $5.0 \mathrm{~g}$ ) using the same procedure for compound $\mathbf{2 a}$. A yellow solid $\mathbf{2 b}$ was obtained $(5.2 \mathrm{~g}, 91 \%) ; \mathrm{mp} 63-64{ }^{\circ} \mathrm{C}$; IR (KBr) 3082, 2984, $1687 \mathrm{~cm}^{-1}$; ${ }^{1} \mathrm{H}$ NMR $\left(200 \mathrm{MHz}, \mathrm{CDCl}_{3}\right): \delta 10.23(\mathrm{~s}, 1 \mathrm{H}), 7.83(\mathrm{~s}, 1 \mathrm{H}), 7.37(\mathrm{~s}, 1 \mathrm{H}), 6.10(\mathrm{~m}$, 1H), 5.49 (dd, J 18.0, $4.8 \mathrm{~Hz}, 1 \mathrm{H}), 5.34$ (dd, J 10.0, $4.4 \mathrm{~Hz}, 1 \mathrm{H}), 4.65$ (dd, J 5.0, $1.4 \mathrm{~Hz}, 2 \mathrm{H}) ;{ }^{13} \mathrm{C} \mathrm{NMR}(50 \mathrm{MHz}$, $\left.\mathrm{CDCl}_{3}\right): \delta 190.8,154.5,137.5,132.9,131.5,120.4,118.5,117.8,112.2,69.9 ; \mathrm{MS}(\mathrm{EI}) \mathrm{m} / \mathrm{z}: 320\left(\mathrm{M}^{+}, 100\right), 279$ (48), 239 (27), 223 (50).

(E)-1-(Allyloxy)-3-(2-nitrovinyl)benzene (3a). To a solution of $2 a$ (12.3 mmol, $2.0 \mathrm{~g}$ ) in glacial AcOH $(20 \mathrm{~mL})$ was added $\mathrm{NH}_{4} \mathrm{OAc}(0.4 \mathrm{~g})$ and nitromethane $(2.0 \mathrm{~mL})$. The solution was heated at reflux with magnetic stirring for $1 \mathrm{~h}$. The mixture was cooled to $\mathrm{rt}$, the resulting dark yellowish precipitate was collected by filtration and was washed with water. The solid was dissolved in $\mathrm{CH}_{2} \mathrm{Cl}_{2}(40.0 \mathrm{~mL})$, filtered through a plug of silica and then the organic phase was dried over anhydrous $\mathrm{Na}_{2} \mathrm{SO}_{4}$ and concentrated under reduced pressure to give a yellowish solid (1.8 g, 70\%); mp 59-60 ${ }^{\circ} \mathrm{C}$; FTIR (KBr): 3112, 3033, 2900, 1628, $1595 \mathrm{~cm}^{-1}$; ${ }^{1} \mathrm{H} \mathrm{NMR}(200 \mathrm{MHz}$, $\left.\mathrm{CDCl}_{3}\right): \delta 7.96(\mathrm{~d}, J 13.6 \mathrm{~Hz}, 1 \mathrm{H}), 7.55(\mathrm{~d}, J 13.6 \mathrm{~Hz}, 1 \mathrm{H}), 7.36(\mathrm{dd}, J$ 9.0, $7.6 \mathrm{~Hz}, 1 \mathrm{H}),(\mathrm{m}, 4 \mathrm{H}), 7.26(\mathrm{td}, J$ J.7, 1.1 $\mathrm{Hz}, 1 \mathrm{H}), 7.00(\mathrm{~m}, 2 \mathrm{H}), 6.06(\mathrm{~m}, 1 \mathrm{H}), 5.43(\mathrm{dd}, J 17.3,3.2 \mathrm{~Hz}, 1 \mathrm{H}), 5.32(\mathrm{dd}, J 10.4,1.4 \mathrm{~Hz}, 1 \mathrm{H}), 4.58(\mathrm{td}, J 5.6,1.5$ $\mathrm{Hz}, 2 \mathrm{H}) ;{ }^{13} \mathrm{C}$ NMR $\left(50 \mathrm{MHz}, \mathrm{CDCl}_{3}\right): \delta$ 158.8, 138.8, 137.1, 132.1, 131.1, 130.2, 121.7, 118.5, 118.0, 114.7, 68.9; MS(EI) $m / z: 205$ (M+, 100), 159 (64), 89 (68), 63 (63).

(E)-1-(allyloxy)-2,4-dibromo-5-(2-nitrovinyl)benzene (3b). Compound $\mathbf{3 b}$ was synthesized from compound $\mathbf{2 b}$ $(9.4 \mathrm{mmol}, 3.0 \mathrm{~g})$ using the same procedure as for compound $2 \mathrm{a}$. A yellow solid was obtained $(3.4 \mathrm{~g}, 70 \%) ; \mathrm{mp}$ 109-110 ${ }^{\circ} \mathrm{C}$; FTIR (KBr): 3103, 2937, 1629, $1577 \mathrm{~cm}^{-1} ;{ }^{1} \mathrm{H}$ NMR (200 MHz, CDCl $): \delta 8.28$ (d, J $\left.13.6 \mathrm{~Hz}, 1 \mathrm{H}\right), 7.86$ (s, 1H), 7.50 (d, J $13.6 \mathrm{~Hz}, 1 \mathrm{H}), 6.99(\mathrm{~s}, 1 \mathrm{H}), 6.05(\mathrm{~m}, 1 \mathrm{H}), 5.49$ (dd, J 17.6, $1.8 \mathrm{~Hz}, 1 \mathrm{H}), 5.38(\mathrm{dd}, J 10.3,1.4 \mathrm{~Hz}$, 
1H), $4.66(t, J 1.4 \mathrm{~Hz}, 1 \mathrm{H}), 4.64(\mathrm{t}, J 1.6 \mathrm{~Hz}, 1 \mathrm{H}) ;{ }^{13} \mathrm{C} \mathrm{NMR}\left(50 \mathrm{MHz}, \mathrm{CDCl}_{3}\right): \delta 154.7,138.6,137.8,136.8,131.3$, 129.6, 118.4, 117.3, 117.0, 111.6, 70.1; MS(EI) m/z: 363 ( $\left.\mathrm{M}^{+}, 75\right), 317(45), 215$ (93), 62 (100).

1-Allyloxy-3-(2-nitro-1-phenylsulfanylethyl)benzene (4a). Compound 3a (32.2 mmol, $6.6 \mathrm{~g})$ was dissolved in $\mathrm{CH}_{2} \mathrm{Cl}_{2}(30.0 \mathrm{~mL})$ and to the solution was added thiophenol $(44.3 \mathrm{mmol}, 4.5 \mathrm{~mL})$ and 4 drops of $\mathrm{N}$ isopropylcyclohexylamine. The resulting solution was stirred for $1 \mathrm{~h}$ at $\mathrm{rt}$. The solution was concentrated and the crude reaction mixture was poured onto silica gel, and then was subjected to flash chromatography using $\mathrm{CH}_{2} \mathrm{Cl}_{2}$ :hexane (20:80) as eluting solvent. Removal of the solvent gave a dark yellow oil (9.3 g, 92\%); FTIR (ATR) 3059, 2916, $1554 \mathrm{~cm}^{-1} ;{ }^{1} \mathrm{H}$ NMR $\left(200 \mathrm{MHz}, \mathrm{CDCl}_{3}\right): \delta 7.33(\mathrm{~m}, 5 \mathrm{H}), 7.20(\mathrm{~m}, 1 \mathrm{H}), 6.81(\mathrm{~m}, 3 \mathrm{H}), 6.03(\mathrm{~m}, 1 \mathrm{H}), 5.38$ $(\mathrm{dt}, J$ 17.0, $1.8 \mathrm{~Hz}, 1 \mathrm{H}), 5.27(\mathrm{dt}, J 8.0,1.6 \mathrm{~Hz}, 1 \mathrm{H}), 4.79(\mathrm{~m}, 3 \mathrm{H}), 4.64(\mathrm{~m}, 2 \mathrm{H}) ;{ }^{13} \mathrm{C} \mathrm{NMR}\left(50 \mathrm{MHz}, \mathrm{CDCl}_{3}\right): \delta$ $158.8,137.8,133.7,133.0,131.9,130.0,129.4,128.8,120.0,117.9,114.9,114.2,78.6,69.0,50.0 ; \mathrm{MS}(\mathrm{EI}) \mathrm{m} / \mathrm{z}$ : $315\left(\mathrm{M}^{+}, 100\right), 269(26), 160(96), 135(70)$.

1-Allyloxy-2,4-dibromo-5-(2-nitro-1-phenylsulfanylethyl)benzene (4b). Compound 4b was synthesized from compound $3 \mathbf{b}(4.3 \mathrm{mmol}, 1.6 \mathrm{~g})$ using the same procedure for compound $4 \mathbf{a}$. A thick yellow liquid was obtained (1.8 g, 90\%); FTIR (ATR): 3294, 3076, 2023, 1648, $1552 \mathrm{~cm}-1 ;{ }^{1} \mathrm{H}$ NMR (500 MHz, CDCl 3 ): $\delta 7.70(\mathrm{~s}$, $1 \mathrm{H}), 7.26(\mathrm{~m}, 5 \mathrm{H}), 6.84(\mathrm{~s}, 1 \mathrm{H}), 5.96(\mathrm{~m}, 1 \mathrm{H}), 5.42(\mathrm{dd}, J$ 16.0, $2.0 \mathrm{~Hz}, 1 \mathrm{H}), 5.34(\mathrm{~m}, 2 \mathrm{H}), 4.70(\mathrm{~m}, 2 \mathrm{H}), 4.45(\mathrm{dt}, J$ 4.0, $1.8 \mathrm{~Hz}, 2 \mathrm{H}) ;{ }^{13} \mathrm{C} \mathrm{NMR}\left(50 \mathrm{MHz}, \mathrm{CDCl}_{3}\right): \delta 154.2,136.8,135.0,133.9,131.6,130.7,129.2,128.9,118.2$, $114.8,113.1,112.4,77.2,69.9,48.5 ; \mathrm{MS}(\mathrm{EI}) \mathrm{m} / z$ : $473\left(\mathrm{M}^{+}, 32\right), 426$ (6), 348 (17), 318 (100).

2-(3-Allyloxyphenyl)-2-phensulfanylethanamine (5a). A stirred mixture of 4 a (31.7 mmol, $10.0 \mathrm{~g})$, zinc (316 $\mathrm{mmol}, 20.6 \mathrm{~g})$ and $\mathrm{AcOH}(80 \mathrm{~mL})$, was cooled in an ice/water bath, and concentrated $\mathrm{HCl}(67 \mathrm{~mL})$ added dropwise and then stirred overnight at rt. The highly acidic solution was then treated with $\mathrm{NH}_{4} \mathrm{OH}(2 \mathrm{~N})$ until the $\mathrm{pH}$ was about 10 . The oily precipitate formed was extracted with $\mathrm{CH}_{2} \mathrm{Cl}_{2}(2 \times 100 \mathrm{~mL})$, the extact dried with $\mathrm{Na}_{2} \mathrm{SO}_{4}$ and concentrated to give the amine as a yellow oil (3.6 g, 40\%). The crude amine was then used in the next step without further purification. FTIR (ATR): 3367, 3064, 2917, 1582, $1479 \mathrm{~cm}^{-1} ;{ }^{1} \mathrm{H} \mathrm{NMR}(200 \mathrm{MHz}$, $\left.\mathrm{CDCl}_{3}\right): \delta 7.34(\mathrm{~m}, 5 \mathrm{H}), 6.83(\mathrm{~m}, 4 \mathrm{H}), 6.10(\mathrm{~m}, 1 \mathrm{H}), 5.36(\mathrm{~d}, J 18.0 \mathrm{~Hz}, 1 \mathrm{H}), 5.24(\mathrm{~d}, J 10.0 \mathrm{~Hz}, 1 \mathrm{H}), 4.47(\mathrm{~m}, 2 \mathrm{H})$, $4.10(\mathrm{t}, J 7.0 \mathrm{~Hz}, 1 \mathrm{H}), 3.08$ (d, J $7.0 \mathrm{~Hz}, 1 \mathrm{H}), 2.01$ (brs, $2 \mathrm{H}) ;{ }^{13} \mathrm{C} \mathrm{NMR}\left(50 \mathrm{MHz}, \mathrm{CDCl}_{3}\right): \delta$ 158.7, 141.7, 134.0, 133.2, 132.3, 129.6, 128.8, 127.2, 120.6, 117.8, 114.5, 113.8, 68.9, 56.5, 46.5; MS(EI) m/z: 285 (M+, 5), 256 (100), 215 (30), 185 (14).

2-(3-Allyloxy-4,6-dibromophenyl)-2-phensulfanylethanamine (5b). To a solution of compound 4b (0.5 g, 1.0 $\mathrm{mmol}$ ) in dry $\mathrm{MeOH}(1.0 \mathrm{~mL})$ was added a $0.1 \mathrm{M}$ solution of $\mathrm{Sml}_{2}(100 \mathrm{~mL}, 10.0 \mathrm{mmol})$ in THF under Ar atmosphere at $\mathrm{rt}$. The mixture was stirred for $24 \mathrm{~h}$ at the same temperature. After this time, it was added dropwise to an aqueous solution of $\mathrm{NaOH}$ until the blue colored suspension turned colorless. The resulting suspension was then extracted with $\mathrm{CH}_{2} \mathrm{Cl}_{2}(3 \times 10 \mathrm{~mL})$. The combined organic extracts were dried with $\mathrm{MgSO}_{4}$, filtered and the solvent was evaporated to afford the crude amine as a clear yellow liquid $(0.4 \mathrm{~g}, 75 \%)$; IR (ATR): 3380, 3064, 2917, 1643, $1578 \mathrm{~cm}^{-1} ;{ }^{1} \mathrm{H}$ NMR (200 MHz, CDCl 3$): \delta 7.70(\mathrm{~s}, 1 \mathrm{H}), 7.22(\mathrm{~m}, 5 \mathrm{H}), 6.96(\mathrm{~s}, 1 \mathrm{H})$, $5.98(\mathrm{~m}, 1 \mathrm{H}), 5.42(\mathrm{~d}, J 16.0 \mathrm{~Hz}, 1 \mathrm{H}), 5.28(\mathrm{~d}, J 10.0 \mathrm{~Hz}, 1 \mathrm{H}), 4.67(\mathrm{t}, J 7.0 \mathrm{~Hz}, 1 \mathrm{H}), 4.51(\mathrm{~m}, 2 \mathrm{H}), 3.05(\mathrm{~d}, J 7.0 \mathrm{~Hz}$, 2H), 2.47 (brs, $2 \mathrm{H}) ;{ }^{13} \mathrm{C} N M R\left(50 \mathrm{MHz}, \mathrm{CDCl}_{3}\right): \delta 154.4,139.3,136.2,133.4,131.9,131.8,128.9,127.4,118.2$, 115.2, 113.5, 111.7, 69.8, 54.9, 46.5; MS(EI) m/z: 443 ( $\left.\mathrm{M}^{+}, 20\right), 414$ (100), 363 (31).

$\mathbf{N}$-(2-(3-Allyloxyphenyl)-2-(phenylthio)ethyl)pent-4-enamide (6a). To an ice cooled solution of amine 5a (1.3 $\mathrm{mmol}, 0.4 \mathrm{~g}$ ) in $\mathrm{CH}_{2} \mathrm{Cl}_{2}(30 \mathrm{~mL}$ ) was added catalytic amounts of DMAP and 4-pentenoyl chloride (1.4 mmol, 0.2 $\mathrm{mL}$ ). The solution was stirred overnight and filtered through a silica plug. Then the solution was concentrated and the residue was purified by circular chromatography using $\mathrm{CH}_{2} \mathrm{Cl}_{2}: \mathrm{MeOH}(97: 3)$ as eluting solvent. Removal of the solvent gave a yellow oil (0.4 g, 75\%); FTIR (ATR): 3064, 2917, 1643, $1539 \mathrm{~cm}^{-1} ;{ }^{1} \mathrm{H}$ NMR (500 $\left.\mathrm{MHz}, \mathrm{CDCl}_{3}\right): \delta 7.34(\mathrm{~m}, 2 \mathrm{H}), 7.22(\mathrm{~m}, 4 \mathrm{H}), 6.82(\mathrm{~m}, 3 \mathrm{H}), 6.00(\mathrm{~m}, 1 \mathrm{H}), 5.73(\mathrm{~m}, 2 \mathrm{H}), 5.39(\mathrm{~d}, J 17.4 \mathrm{~Hz}, 1 \mathrm{H}), 5.27$ (d, J $10.9 \mathrm{~Hz}, 1 \mathrm{H}), 4.98(\mathrm{~d}, J 15.0 \mathrm{~Hz}, 1 \mathrm{H}), 4.94(\mathrm{~d}, J 10.0 \mathrm{~Hz}, 1 \mathrm{H}), 4.48(\mathrm{~m}, 2 \mathrm{H}), 4.33(\mathrm{t}, J 7.4 \mathrm{~Hz}, 1 \mathrm{H}), 3.75(\mathrm{~m}$, 
1H), $3.60(\mathrm{~m}, 1 \mathrm{H}), 2.29(\mathrm{~m}, 2 \mathrm{H}), 2.16(\mathrm{t}, J 7.8 \mathrm{~Hz}, 2 \mathrm{H}) ;{ }^{13} \mathrm{C} \mathrm{NMR}\left(125 \mathrm{MHz}, \mathrm{CDCl}_{3}\right): \delta$ 172.5, 159.0, 141.1, 137.1, 134.1, 133.3, 132.1, 129.9, 129.1, 127.5, 120.6, 117.9, 115.7, 114.5, 114.3, 68.9, 52.3, 44.4, 35.8, 29.6; MS(EI) m/z: $367\left(\mathrm{M}^{+}, 10\right), 268(100), 258(66), 176(78)$. HRMS(FAB) calcd for $\mathrm{C}_{22} \mathrm{H}_{25} \mathrm{NO}_{2} \mathrm{~S}(\mathrm{M}+\mathrm{H})^{+}$368.1684. Found 368.1699.

$\mathbf{N}$-(2-(5-allyloxy-2,4-dibromophenyl)-2-(phenylthio)ethyl)pent-4-enamide $\quad$ (6b). Compound $6 \mathrm{~b}$ was synthesized from $\mathbf{5 b}(1.5 \mathrm{mmol}, 0.7 \mathrm{~g})$, using the same procedure as for compound $\mathbf{6 a}$. A dark yellow liquid was obtained (0.4 g, 57\%); FTIR (neat) 3289, 3073, 2928, 1647, 1545, 1467, $1245 \mathrm{~cm}^{-1} ;{ }^{1} \mathrm{H} \mathrm{NMR}(500 \mathrm{MHz} \mathrm{CDCl}): \delta$ $7.70(\mathrm{~s}, 1 \mathrm{H}), 7.26(\mathrm{~m}, 5 \mathrm{H}), 6.34(\mathrm{~s}, 1 \mathrm{H}), 5.99(\mathrm{~m}, 1 \mathrm{H}) ; 5.20(\mathrm{~m}, 2 \mathrm{H}), 5.47(\mathrm{dd}, J$ 16.0, $4.0 \mathrm{~Hz}, 1 \mathrm{H}), 5.30$ (dd, J 9.0, $4.0 \mathrm{~Hz}, 1 \mathrm{H}), 5.03(\mathrm{dd}, J$ 15.0, $4.0 \mathrm{~Hz}, 1 \mathrm{H}), 4.95(\mathrm{~m}, 1 \mathrm{H}), 4.78(\mathrm{t}, J 7.0, \mathrm{~Hz}, 1 \mathrm{H}), 4.49(\mathrm{~m}, 2 \mathrm{H}), 2.70(\mathrm{~m}, 2 \mathrm{H}), 2.38$ $(\mathrm{m}, 4 \mathrm{H}) ;{ }^{13} \mathrm{C}$ NMR $\left(125 \mathrm{MHz}, \mathrm{CDCl}_{3}\right): \delta 171.8,154.2,138.1,136.4,136.1,132.3,132.2,131.7,128.8,127.6$, 117.9, 115.4, 115.0, 113.1, 111.8, 69.7, 50.7, 42.9, 35.6, 29.3; MS(EI) m/z: 525 (M+, 12), 446 (85), 416 (100), 334 (78).

N-(2-(3-allyloxyphenyl)-2-(phenylthio)ethyl)hex-5-enamide 7a. To a well stirred and ice cooled solution of DCC (7.7 mmol, $1.6 \mathrm{~g})$, under argon, DMAP (0.05 g) and 5-hexenoic acid (7.7 mmol, $0.9 \mathrm{~mL})$ in dry THF (100 mL) was added the amine $5 \mathrm{a}(7.7 \mathrm{mmol}, 2.2 \mathrm{~g})$. The mixture was strirred at $\mathrm{rt}$ for $24 \mathrm{~h}$ then it was filtered, dried with $\mathrm{Na}_{2} \mathrm{SO}_{4}$ and concentrated. The residue was purified using circular chromatography eluting with $\mathrm{CH}_{2} \mathrm{Cl}_{2} / \mathrm{MeOH}$ (97:3) to afford the product as a yellow liquid (2.4 g, 80\%); FTIR (neat): 3293, 3073, 2926, 1644 $\mathrm{cm}^{-1} ;{ }^{1} \mathrm{H}$ NMR $\left(400 \mathrm{MHz}, \mathrm{CDCl}_{3}\right): \delta 7.33(\mathrm{~m}, 2 \mathrm{H}), 7.21(\mathrm{~m}, 4 \mathrm{H}), 6.83(\mathrm{~m}, 3 \mathrm{H}), 6.03(\mathrm{~m}, 1 \mathrm{H}), 5.71(\mathrm{~m}, 2 \mathrm{H}), 5.39(\mathrm{~d}$, J $16.0 \mathrm{~Hz}, 1 \mathrm{H}), 5.27(\mathrm{~d}, J 11.0 \mathrm{~Hz}, 1 \mathrm{H}), 4.95(\mathrm{~m}, 2 \mathrm{H}), 4.48(\mathrm{~m}, 2 \mathrm{H}), 4.34(\mathrm{t}, J 8.0 \mathrm{~Hz}, 1 \mathrm{H}), 3.75(\mathrm{~m}, 1 \mathrm{H}), 3.61$ $(\mathrm{m}, 1 \mathrm{H}), 2.06(\mathrm{~m}, 2 \mathrm{H}), 2.00(\mathrm{~m}, 2 \mathrm{H}), 1.65(\mathrm{~m}, 2 \mathrm{H}) ;{ }^{13} \mathrm{C} \mathrm{NMR}\left(50 \mathrm{MHz}, \mathrm{CDCl}_{3}\right): \delta 172.8,158.8,140.9,137.8,134.0$, 133.1, 131.9, 129.7, 128.9, 127.3, 120.4, 117.7, 115.3, 114.3, 114.1, 68.8, 52.2, 44.2, 35.7, 33.0, 24.6; MS(EI) m/z: $381\left(\mathrm{M}^{+}, 12\right), 268$ (100), 255 (55), 176 (78); HRMS(FAB) Calcd for $\mathrm{C}_{23} \mathrm{H}_{27} \mathrm{~N}_{2} \mathrm{OS}(\mathrm{M}+\mathrm{H})^{+}$382.18407. Found 382.18410 .

N-(2-(3-allyloxyphenyl)-2-(phenylthio)ethyl)hept-6-enamide 8a. Compound 8a was synthesized from the amine $5 \mathrm{a}(7.0 \mathrm{mmol}, 2.0 \mathrm{~g})$ using the procedure for $7 \mathrm{a}$ and $0.95 \mathrm{~mL}$ of 6-heptenoic acid $(7.0 \mathrm{mmol}, 0.9 \mathrm{~g})$. A dark yellow liquid was obtained (2.3 g, 82\%); FTIR (neat): 3295, 3064, 2927, $1644 \mathrm{~cm}^{-1} ;{ }^{1} \mathrm{H} \mathrm{NMR}(600 \mathrm{MHz}$, $\left.\mathrm{CDCl}_{3}\right): \delta 7.33(\mathrm{~m}, 2 \mathrm{H}), 7.22(\mathrm{~m}, 4 \mathrm{H}), 6.82(\mathrm{~m}, 3 \mathrm{H}), 6.03(\mathrm{~m}, 1 \mathrm{H}), 5.75(\mathrm{~m}, 1 \mathrm{H}), 5.59(\mathrm{~s}, 1 \mathrm{H}), 5.39(\mathrm{dd}, J 17.0,2.0$ $\mathrm{Hz}, 1 \mathrm{H}), 5.27(\mathrm{dd}, J 10.0,2.0 \mathrm{~Hz}, 1 \mathrm{H}), 4.97(\mathrm{dd}, J 17.0,2.0 \mathrm{~Hz}, 1 \mathrm{H}), 4.92(\mathrm{dd}, J 11.0,2.0 \mathrm{~Hz}, 1 \mathrm{H}), 4.49(\mathrm{~m}, 2 \mathrm{H})$, $4.34(\mathrm{t}, J 8.0 \mathrm{~Hz}, 1 \mathrm{H}), 3.77(\mathrm{~m}, 1 \mathrm{H}), 3.61(\mathrm{~m}, 1 \mathrm{H}), 2.07(\mathrm{t}, J 7.0 \mathrm{~Hz}, 2 \mathrm{H}), 2.02(\mathrm{~m}, 2 \mathrm{H}), 1.55(\mathrm{~m}, 2 \mathrm{H}), 1.35(\mathrm{~m}, 2 \mathrm{H})$; ${ }^{13} \mathrm{C} \mathrm{NMR}\left(150 \mathrm{MHz}, \mathrm{CDCl}_{3}\right): \delta 172.9,158.9,140.9,138.4,134.0,133.1,131.9,129.7,128.9,127.3,120.4,117.7$, 114.7, 114.3, 114.2, 68.8, 52.2, 44.2, 36.5, 33.4, 28.4, 25.0; MS(EI) m/z: 395 (M+, 13), 286 (50), 268 (100), 176 (80).

[2-(5-allyloxy-2,4-dibromophenyl)-2-(phenylsulfanyl)ethyl]-6-heptenamide $\quad$ (8b). Compound $8 \mathbf{b}$ was synthesized from $5 \mathbf{b}(1.5 \mathrm{mmol}, 0.7 \mathrm{~g})$, using the procedure for compound 7a. A dark yellow liquid was obtained (0.4 g, 57\%); FTIR (neat): 3291, 3077, 2929, $1649 \mathrm{~cm}^{-1} ;{ }^{1} \mathrm{H} \mathrm{NMR}\left(600 \mathrm{MHz}, \mathrm{CDCl}_{3}\right): \delta 7.71(\mathrm{~s}, 1 \mathrm{H}), 7.30$ $(\mathrm{m}, 5 \mathrm{H}), 6.86(\mathrm{~s}, 1 \mathrm{H}), 6.00(\mathrm{~m}, 1 \mathrm{H}), 5.75(\mathrm{~m}, 1 \mathrm{H}), 5.55(\mathrm{~s}, 1 \mathrm{H}), 5.45(\mathrm{dd}, J 17.0,2.0 \mathrm{~Hz}, 1 \mathrm{H}), 5.30(\mathrm{dd}, J 10.0,2.0$ $\mathrm{Hz}, 1 \mathrm{H}), 4.97(\mathrm{dd}, J=17.0,2.0 \mathrm{~Hz}, 1 \mathrm{H}), 4.93(\mathrm{dd}, J 10.0,2.0 \mathrm{~Hz}, 1 \mathrm{H}), 4.78(\mathrm{t}, J 8.0 \mathrm{~Hz}, 1 \mathrm{H}), 4.49(\mathrm{~m}, 2 \mathrm{H}), 3.76(\mathrm{~m}$, $1 \mathrm{H}), 3.65(\mathrm{~m}, 1 \mathrm{H}), 2.08(\mathrm{~m}, 2 \mathrm{H}), 2.01(\mathrm{~m}, 2 \mathrm{H}), 1.55(\mathrm{~m}, 4 \mathrm{H}), 1.32(\mathrm{~m}, 2 \mathrm{H}) ;{ }^{13} \mathrm{C} \mathrm{NMR}\left(50 \mathrm{MHz}, \mathrm{CDCl}_{3}\right): \delta 172.8$, 154.6, 138.5, 138.4, 136.4, 132.7, 132.6, 132.1, 129.05, 127.9, 118.2, 115.3, 114.7, 113.5, 112.2, 69.9, 50.8, 43.1, 36.5, 33.5, 28.4, 24.9. MS(EI) m/z: $554\left(\mathrm{M}^{+}, 6\right), 474$ (42), 444 (100), 426 (60); HRMS(FAB) calcd for $\mathrm{C}_{24} \mathrm{H}_{28} \mathrm{Br}_{2} \mathrm{NO}_{2} \mathrm{~S}(\mathrm{M}+\mathrm{H})^{+} 552.02075$. Found 552.02080.

11-phenylsulfanyl-2-oxa-9-azabicyclo[10.3.1]hexadeca-1(16),4E,12,14-tetraen-8-one (9a). To solution of diene $6 a(80.0 \mathrm{mg}, 0.2 \mathrm{mmol})$ in $55 \mathrm{~mL}$ of dry $\mathrm{CH}_{2} \mathrm{Cl}_{2}(0.004 \mathrm{M})$ was added the Hoveyda-Grubbs catalyst $(9.0$ $\mathrm{mg}, 0.014 \mathrm{mmol}$ ) and refluxed for $12 \mathrm{~h}$. The reaction was carried out in an open system and to remove 
ethylene from the system a very gentle flow of argon was used. Then, the reaction mixture was filtered through a short fluorisil pad to remove the catalyst residues. The solvent was evaporated under reduced pressure and the crude product was purified using circular chromatography to give the macrocycle product (53.4 mg, 73\% yield). Mp 146-148 ${ }^{\circ} \mathrm{C}$; FTIR (KBr): 3292, 3062, 2922, 1655, 1635, $1534 \mathrm{~cm}^{-1} ;{ }^{1} \mathrm{H}$ NMR $(500 \mathrm{MHz}$, $\left.\mathrm{CDCl}_{3}\right)$ : $7.41(\mathrm{dt}, J$ 6.5, $1.4 \mathrm{~Hz}, 2 \mathrm{H}), 7.24(\mathrm{~m}, 4 \mathrm{H}), 6.94(\mathrm{dt}, J$ 7.5, $1.0 \mathrm{~Hz}, 1 \mathrm{H}), 6.82(\mathrm{dd}, J 8.1,1.8 \mathrm{~Hz}, 1 \mathrm{H}), 6.76(\mathrm{t}, J$ $1.9 \mathrm{~Hz}, 1 \mathrm{H}), 5.62(\mathrm{~m}, 1 \mathrm{H}) ; 5.51(\mathrm{dt}, J 16.0,5.0 \mathrm{~Hz}, 1 \mathrm{H}), 5.45(\mathrm{brs}, 1 \mathrm{H}), 4.64(\mathrm{~m}, 2 \mathrm{H}), 4.50(\mathrm{dd}, J 8.7,4.1 \mathrm{~Hz}, 1 \mathrm{H})$, $4.05(\mathrm{~m}, 1 \mathrm{H}), 3.14(\mathrm{~m}, 1 \mathrm{H}), 2.48(\mathrm{~m}, 1 \mathrm{H}), 2.35(\mathrm{~m}, 1 \mathrm{H}), 2.27(\mathrm{~m}, 1 \mathrm{H}), 2.04(\mathrm{~m}, 1 \mathrm{H}) ;{ }^{13} \mathrm{C} \mathrm{NMR}\left(50 \mathrm{MHz}, \mathrm{CDCl}_{3}\right): \delta$ 171.8, 157.1, 139.6, 134.1, 133.9, 131.5, 130.0, 129.1, 127.8, 127.3, 119.6, 117.9, 116.9, 69.1, 51.0, 44.9, 37.2, 27.7; MS(EI) m/z: 339 (M+, 33), 230 (100), 136 (45); HRMS(FAB) calcd for $\mathrm{C}_{20} \mathrm{H}_{22} \mathrm{NO}_{2} \mathrm{~S}(\mathrm{M}+\mathrm{H})^{+} 340.13712$. Found 340.13716.

13,15-Dibromo-11-phenylsulfanyl-2-oxa-9-azabicyclo[10.3.1]hexadeca-(16),4E,12,14-tetraen-8-one

(9b). Compound 9b was synthesized using the same procedure for compound 9a using diene $6 \mathbf{b}$ (55.2 mg, 0.1 mmol). A gray solid was obtained (37.4 mg, 72\%); FTIR (KBr): 3281, 3064, 2926, 1648, $1544 \mathrm{~cm}^{-1}$; ${ }^{1} \mathrm{H}$ NMR (500 $\left.\mathrm{MHz}_{\mathrm{CDCl}}\right): \delta 7.84(\mathrm{~s}, 1 \mathrm{H}), 7.40(\mathrm{~m}, 2 \mathrm{H}), 7.29(\mathrm{~m}, 3 \mathrm{H}), 6.72(\mathrm{~s}, 1 \mathrm{H}), 5.69(\mathrm{~m}, 1 \mathrm{H}), 5.71(\mathrm{dt}, J 16.0,5.0 \mathrm{~Hz}, 1 \mathrm{H})$, 5.43 (brs, 1H), $4.49\left(\mathrm{t}, J\right.$ 8.0, 1H), $4.65(\mathrm{~m}, 2 \mathrm{H}), 4.02(\mathrm{~m}, 1 \mathrm{H}), 4.52(\mathrm{~m}, 1 \mathrm{H}), 2.03(\mathrm{~m}, 4 \mathrm{H}) ;{ }^{13} \mathrm{C} \mathrm{NMR}(125 \mathrm{MHz}$, $\left.\mathrm{CDCl}_{3}\right): \delta 171.8,156.2,142.1,136.4,135.2,130.1,129.9128 .1128 .0127 .2,117.2,116.2,112.8,70.2,50.80$, 45.2, 38.3, 38.1; MS(EI) m/z: $497\left(\mathrm{M}^{+}, 55\right), 388$ (100), 355 (12).

12-Phenylsulfanyl-2-oxa-10-azabicyclo[11.3.1] heptadeca-1(17),4E,13,15-tetraen-9-one (10a). Compound 10a was synthesized using the same procedure as for compound 9a using diene 7a (2.6 mmol, $1.0 \mathrm{~g})$. A gray solid was obtained $(0.5 \mathrm{~g}, 52 \%)$; mp 158-160 ${ }^{\circ} \mathrm{C}$; FTIR (KBr): 3298, 3056, 2928, 1648, $1595 \mathrm{~cm}^{-1} ;{ }^{1} \mathrm{H} \mathrm{NMR}(500 \mathrm{MHz}$, $\left.\mathrm{CDCl}_{3}\right): \delta 7.45(\mathrm{~m}, 2 \mathrm{H}), 7.25(\mathrm{~m}, 4 \mathrm{H}), 7.04(\mathrm{~d}, J 7.6 \mathrm{~Hz}, 1 \mathrm{H}), 6.80(\mathrm{dd}, J 8.2,2.4 \mathrm{~Hz}, 1 \mathrm{H}), 6.64(\mathrm{t}, J 1.7 \mathrm{~Hz}, 1 \mathrm{H}), 5.70$ $(\mathrm{m}, 1 \mathrm{H}), 5.46(\mathrm{~m}, 1 \mathrm{H}), 5.32$ (brs, 1H), $4.69(\mathrm{dd}, J 10.2,4.3 \mathrm{~Hz}, 1 \mathrm{H}), 4.62(\mathrm{~m}, 2 \mathrm{H}), 4.00(\mathrm{~m}, 1 \mathrm{H}), 3.10(\mathrm{~m}, 1 \mathrm{H}), 2.29$ $(\mathrm{m}, 2 \mathrm{H}), 2.10(\mathrm{~m}, 1 \mathrm{H}), 1.96(\mathrm{~m}, 1 \mathrm{H}), 1.84(\mathrm{~m}, 1 \mathrm{H}), 1.70(\mathrm{~m}, 1 \mathrm{H}) ;{ }^{13} \mathrm{C} \mathrm{NMR}\left(125 \mathrm{MHz}, \mathrm{CDCl}_{3}\right): \delta 173.1,157.6$, 140.0, 134.4, 134.3, 131.1, 130.0, 129.1, 127.8, 127.0, 119.2, 117.1, 113.8, 67.8, 49.8, 45.5, 34.4, 33.9, 20.9; MS(EI) $m / z: 353\left(\mathrm{M}^{+}, 50\right), 244$ (100), 228 (32); HRMS(FAB) calcd for $\mathrm{C}_{21} \mathrm{H}_{24} \mathrm{NO}_{2} \mathrm{~S}(\mathrm{M}+\mathrm{H})^{+}$354.15277. Found 354.15290.

(E)-13-(phenylthio)-2-oxa-11-aza-1(1,3)-benzenacyclotridecaphan-4-en-10-one (11a). Compound 11a was synthesized using the same procedure as for compound 9a using diene $8 \mathrm{a}$ ( $308 \mathrm{mg}, 0.78 \mathrm{mmol}$ ) to give brown crystals (204 mg, $71 \%$ yield); mp 118-121 ${ }^{\circ} \mathrm{C}$; FTIR (KBr) 3287, 3056, 2930, 1646, $1594 \mathrm{~cm}^{-1} ;{ }^{1} \mathrm{H} \mathrm{NMR}(600 \mathrm{MHz}$, $\left.\mathrm{CDCl}_{3}\right): \delta 7.43(\mathrm{~m}, 2 \mathrm{H}), 7.27(\mathrm{~m}, 4 \mathrm{H}), 7.21(\mathrm{t}, J 7.8 \mathrm{~Hz}, 1 \mathrm{H}), 6.96(\mathrm{~d}, J 8.0 \mathrm{~Hz}, 1 \mathrm{H}), 6.87(\mathrm{dd}, J 8.0,4.0 \mathrm{~Hz}, 1 \mathrm{H}), 6.70$ (t, J $1.8 \mathrm{~Hz}, 1 \mathrm{H}), 5.63(\mathrm{~m}, 1 \mathrm{H}), 5.47(\mathrm{~m}, 1 \mathrm{H}), 5.23(\mathrm{brs}, 1 \mathrm{H}), 4.65(\mathrm{~m}, 1 \mathrm{H}), 4.60(\mathrm{~m}, 1 \mathrm{H}) 4.29(\mathrm{dd}, J 10.0,4.0 \mathrm{~Hz}$, $1 \mathrm{H}), 4.16(\mathrm{~m}, 1 \mathrm{H}), 3.24(\mathrm{~m}, 1 \mathrm{H}), 2.30(\mathrm{~m}, 1 \mathrm{H}), 2.0(\mathrm{~m}, 2 \mathrm{H}), 1.44(\mathrm{~m}, 2 \mathrm{H}), 1.30(\mathrm{~m}, 2 \mathrm{H}) ;{ }^{13} \mathrm{C} \mathrm{NMR}\left(50 \mathrm{MHz}, \mathrm{CDCl}_{3}\right)$ : $\delta 173.3,158.2,140.2,134.6,134.3,131.4,130.4,129.0,127.1,126.7,119.1,117.6,115.7,68.6,50.9,44.3$, 36.6, 31.5, 27.6, 25.6; EM(IE) m/z: $367\left(\mathrm{M}^{+}, 10\right), 258$ (100), 228 (37). HRMS(FAB) calcd for $\mathrm{C}_{22} \mathrm{H}_{26} \mathrm{NO}_{2} \mathrm{~S}(\mathrm{M}+\mathrm{H})^{+}$ 368.16842. Found 368.16847.

15,17-Dibromo-13-phenylsulfanyl-2-oxa-11-azabicyclo[12.3.1]octadeca-1-(18),4,14,16-tetraen-10-one (11b). To solution of diene $8 \mathbf{b}(118.4 \mathrm{mg}, 0.214 \mathrm{mmol})$ in $215 \mathrm{~mL}$ of dry $\mathrm{CH}_{2} \mathrm{Cl}_{2}(0.001 \mathrm{M})$ was added the HoveydaGrubb's catalyst $(6.7 \mathrm{mg}, 0.011 \mathrm{mmol})$ and the mioxture heated at reflux for $12 \mathrm{~h}$. The reaction was carried out in an open system and to remove ethylene from the system a very gentle flow of Ar was used. Then, the reaction mixture was filtered through a short fluorisil pad to remove the catalyst residues. The solvent was evaporated under reduced pressure and the crude product was purified using circular chromatography to give the macrocyclic product (113 mg, 80\% yield). FTIR (ATR): 3292, 3059, 2930, 2859, 1651, $1551 \mathrm{~cm}^{-1} ;{ }^{1} \mathrm{H} \mathrm{NMR}$ $\left(500 \mathrm{MHz}, \mathrm{CDCl}_{3}\right): \delta 7.74(\mathrm{~s}, 1 \mathrm{H}), 7.43(\mathrm{~m}, 2 \mathrm{H}), 7.31(\mathrm{~s}, 3 \mathrm{H}), 6.64(\mathrm{~s}, 1 \mathrm{H}), 5.70(\mathrm{~m}, 1 \mathrm{H}), 5.73(\mathrm{dt}, J 16.0,5.0 \mathrm{~Hz}$, $1 \mathrm{H}), 5.67($ brs, $1 \mathrm{H}), 4.80(\mathrm{t}, J 8.0 \mathrm{~Hz}, 1 \mathrm{H}), 4.67(\mathrm{~m}, 2 \mathrm{H}), 4.20(\mathrm{~m}, 1 \mathrm{H}), 3.4(\mathrm{~m}, 1 \mathrm{H}), 2.02(\mathrm{~m}, 4 \mathrm{H}), 1.40(\mathrm{~m}, 2 \mathrm{H})$, 
1.23 (m, 2H); ${ }^{13} \mathrm{C}$ NMR (125 MHz, $\left.\mathrm{CDCl}_{3}\right): \delta$ 173.0, 138.3, 137.2, 137.0, 135.4, 133.2, 131.9, 131.3, 129.2, 129.1, 127.6, 127.4, 126.5, 125.9, 70.2, 51.2, 40.9, 36.2, 31.2, 26.5, 24.7, 19.9; MS(EI) m/z: 525 (M+, 60), 414 (100), 381 (12); MS(ESI) m/z: $526(\mathrm{M}+\mathrm{H})^{+}$; HRMS(FAB) calcd for $\mathrm{C}_{22} \mathrm{H}_{24} \mathrm{Br}_{2} \mathrm{NO}_{2} \mathrm{~S}(\mathrm{M}+\mathrm{H})^{+}$523.98945. Found 523.98990.

2-Oxa-9-azabicyclo[10.3.1] hexadeca-1(16),4E,11Z(10),12,14-penten-8-one (12a). To a solution of sulfide 9a $(70 \mathrm{mg}, 0.21 \mathrm{mmol})$ in $\mathrm{MeOH}(5.0 \mathrm{~mL})$ were added dropwise $5.0 \mathrm{~mL}$ of an aqueous solution of sodium periodate $(88.3 \mathrm{mg}, 0.42 \mathrm{mmol})$ at $\mathrm{rt}$ and the mixture stirred for $2 \mathrm{~h}$. The mixture was extracted with $\mathrm{CH}_{2} \mathrm{Cl}_{2}$ $(3 \times 5 \mathrm{~mL})$ and the combined organic phase was dried with $\mathrm{Na}_{2} \mathrm{SO}_{4}$, filtered and the solvent was evaporated to give the sulfoxide as a colorless oil. To a solution of sulfoxide in toluene $(20 \mathrm{~mL})$ was added $\mathrm{K}_{2} \mathrm{CO}_{3}(189 \mathrm{mg}, 1.4$ $\mathrm{mmol})$ and stirred at reflux for $3 \mathrm{~h}$. The solvent was evaporated and the residue was dissolved with $\mathrm{CH}_{2} \mathrm{Cl}_{2}(20$ $\mathrm{mL}$ ) and washed with brine. The organic phase was dried with $\mathrm{Na}_{2} \mathrm{SO}_{4}$, filtered and the solvent was evaporated to give a crude product that was purified by circular chromatography to give a colorless oil ( $44.6 \mathrm{mg}, 94 \%$ yield); FTIR (KBr) 3292, 3062, 2922, 1655, 1635, $1534 \mathrm{~cm}^{-1} ;{ }^{1} \mathrm{H}$ NMR $\left(500 \mathrm{MHz}, \mathrm{CDCl}_{3}\right): 7.91(\mathrm{~d}, J 10.0 \mathrm{~Hz}, 1 \mathrm{H})$, $7.24(\mathrm{~m}, 1 \mathrm{H}), 6.96(\mathrm{dd}, J$ 11.0, $9.0 \mathrm{~Hz}, 1 \mathrm{H}), 6.84(\mathrm{~m}, 2 \mathrm{H}), 6.74(\mathrm{~d}, J 8.0 \mathrm{~Hz}, 1 \mathrm{H}), 5.87(\mathrm{~m}, 1 \mathrm{H}), 5.83(\mathrm{~d}, J 10.0 \mathrm{~Hz}$, $1 \mathrm{H}) ; 5.67(\mathrm{dt}, J 16.0,5.0 \mathrm{~Hz}, 1 \mathrm{H}), 4.78(\mathrm{dd}, J 5.0,1.0 \mathrm{~Hz}, 2 \mathrm{H}), 2.5(\mathrm{~m}, 2 \mathrm{H}), 2.47(\mathrm{~m}, 2 \mathrm{H}) ;{ }^{13} \mathrm{C} \mathrm{NMR}(50 \mathrm{MHz}$, $\left.\mathrm{CDCl}_{3}\right): \delta 169.6,157.2,135.7,134.0,130.9,130.2,122.7,120.8,116.9,114.9,111.1,69.4,35.9,26.9 ; \mathrm{MS}(\mathrm{EI})$ $\mathrm{m} / \mathrm{z}: 229\left(\mathrm{M}^{+}, 50\right), 216$ (35), 159 (100); HRMS(FAB) calcd for $\mathrm{C}_{14} \mathrm{H}_{15} \mathrm{NO}_{2}(\mathrm{M}+\mathrm{H})^{+}$230.1103. Found 230.1099.

13,15-Dibromo-2-oxa-9-azabicyclo[10.3.1] hexadeca-1(16),4E,11Z(10),12,14-penten-8-one (12b). Compound $12 \mathrm{~b}$ was synthesized from compound $\mathbf{9 b}(37.4 \mathrm{mg}, 0.075 \mathrm{mmol})$ using the same procedure for compound $12 \mathrm{a}$. Colorless oil was obtained (29.0 mg, 99.7\%); FTIR (KBr) 3280, 3050, 2926, 1648, $1544 \mathrm{~cm}^{-1} ;{ }^{1} \mathrm{H} \mathrm{NMR}(500 \mathrm{MHz}$, $\mathrm{CDCl}_{3}$ ): $\delta 7.90$ (brs, $\left.1 \mathrm{H}\right), 7.80(\mathrm{~s}, 1 \mathrm{H}), 7.30(\mathrm{dd}, J 10.0,9.0 \mathrm{~Hz}, 1 \mathrm{H}), 6.50(\mathrm{~s}, 1 \mathrm{H}), 6.18(\mathrm{~d}, J 10.0 \mathrm{~Hz}, 1 \mathrm{H}), 5.70(\mathrm{~m}$, $1 \mathrm{H}), 5.60(\mathrm{dt}, J 16.0,5.0 \mathrm{~Hz}, 1 \mathrm{H}), 4.61(\mathrm{~d}, J 5.0 \mathrm{~Hz}, 2 \mathrm{H}), 2.22(\mathrm{~m}, 4 \mathrm{H}) ;{ }^{13} \mathrm{C} \mathrm{NMR}\left(125 \mathrm{MHz}, \mathrm{CDCl}_{3}\right): \delta 171.8,156.2$, 136.4, 135.2, 129.3 128.5, 124.5, 121.2, 113.2, 112.8, 106.7, 70.2, 38.3, 38.1; MS(EI) m/z: 387 (M+, 58). 307 (43), 253 (100); $\mathrm{HRMS}(\mathrm{FAB})$ calcd for $\mathrm{C}_{14} \mathrm{H}_{13} \mathrm{Br}_{2} \mathrm{NO}_{2}(\mathrm{M}+\mathrm{H})^{+}$385.9313. Found 385.9309.

2-Oxa-10-azabicyclo[11.3.1] heptadeca-1(17),4E,12Z(11),13,15-penten-9-one (13a). Compound 13a was synthesized from compound $10 \mathrm{a}(17.0 \mathrm{mg}, 0.05 \mathrm{mmol})$ using the same procedure as for compound 12a. A colorless oil was obtained (11.0 mg, 94\%); FTIR (KBr): 3281, 3056, 2926, 1650, $1596 \mathrm{~cm}^{-1} ;{ }^{1} \mathrm{H} \mathrm{NMR}(500 \mathrm{MHz}$, $\mathrm{CDCl}_{3}$ ): 7.50 (brs, 1H), $7.24(\mathrm{t}, J 8.0 \mathrm{~Hz}, 1 \mathrm{H}), 6.88$ (brs, 1H), $6.86(\mathrm{dd}, J 11.0,9.0 \mathrm{~Hz}, 1 \mathrm{H}), 6.81(\mathrm{~m}, 1 \mathrm{H}), 6.73(\mathrm{~d}, J$ $8.0 \mathrm{~Hz}, 1 \mathrm{H}), 5.88(\mathrm{~d}, J 10.0 \mathrm{~Hz}, 1 \mathrm{H}), 5.81(\mathrm{dt}, J 16.0,5.0 \mathrm{~Hz}, 1 \mathrm{H}), 5.68(\mathrm{~m}, 1 \mathrm{H}), 4.68(\mathrm{dd}, J 6.0,1.0 \mathrm{~Hz}, 2 \mathrm{H}), 2.27$ $(\mathrm{m}, 4 \mathrm{H}), 1.92(\mathrm{~m}, 2 \mathrm{H}) .{ }^{13} \mathrm{C} \mathrm{NMR}\left(125 \mathrm{MHz}, \mathrm{CDCl}_{3}\right): \delta 171.3,158.2,136.7,134.0,130.3,129.4,122.8,120.5$, 116.6, 112.0, 111.7, 68.3, 36.5, 34.4, 24.3; MS(EI) $\mathrm{m} / \mathrm{z}: 243\left(\mathrm{M}^{+}, 50\right), 135$ (100); HRMS(FAB) calcd for $\mathrm{C}_{15} \mathrm{H}_{17} \mathrm{NO}_{2}$ $(\mathrm{M}+\mathrm{H})^{+}$244.1259. Found 244.1255.

2-Oxa-11-aza-bicyclo[12.3.1] octadeca-1(18),4E,13Z(12),14,16-penten-10-one (14a). Compound 14a was synthesized from compound $11 \mathrm{a}(204 \mathrm{mg}, 0.6 \mathrm{mmol}$ ) using the same procedure as for compound 12a. A colorless oil was obtained (86.3 mg, 60\%); FTIR (ATR): 3287, 3056, 2930, 1646, $1594 \mathrm{~cm}^{-1} ;{ }^{1} \mathrm{H} \mathrm{NMR}(600 \mathrm{MHz}$, $\mathrm{CDCl}_{3}$ ): $\delta 7.83$ (brs, $\left.1 \mathrm{H}\right), 7.24(\mathrm{t}, J 8.0 \mathrm{~Hz}, 1 \mathrm{H}), 6.95$ (dd, J 11.0, $\left.9.0 \mathrm{~Hz}, 1 \mathrm{H}\right), 6.83$ (brs, 2H), $6.72(\mathrm{dd}, J 8.0,4.0 \mathrm{~Hz}$, $1 \mathrm{H}), 5.90(\mathrm{~d}, J 10.0 \mathrm{~Hz}, 1 \mathrm{H}), 5.83(\mathrm{~m}, 1 \mathrm{H}), 5.69(\mathrm{~m}, 1 \mathrm{H}), 4.79(\mathrm{~d}, J 5.0 \mathrm{~Hz}, 2 \mathrm{H}), 2.03(\mathrm{~m}, 4 \mathrm{H}), 1.43(\mathrm{~m}, 2 \mathrm{H}), 1.3(\mathrm{~m}$, $2 \mathrm{H}) ;{ }^{13} \mathrm{C}$ NMR $\left(150 \mathrm{MHz}, \mathrm{CDCl}_{3}\right): \delta 173.3,158.2,136.0,132.3,129.0,128.9,120.7,119.1,113.6,113.0,110.4$, 75.7, 39.3, 28.7, 27.6, 25.6; MS(EI) m/z: $257\left(\mathrm{M}^{+}, 100\right), 228$ (25), 215 (16). HRMS(FAB) calcd for $\mathrm{C}_{16} \mathrm{H}_{19} \mathrm{NO}_{2}$ $(\mathrm{M}+\mathrm{H})^{+}$258.1416. Found 258.1421 .

15,17-Dibromo-2-oxa-11-azabicyclo[12.3.1]octadeca-1(18),4E,13Z(12),14,16-penten-10-one 14b. Compound 14b was synthesized from compound $\mathbf{1 1 b}(113 \mathrm{mg}, 0.2 \mathrm{mmol})$ using the same procedure as for compound $12 \mathrm{a}$. A colorless oil was obtained (41.1 mg, 45\%); FTIR (KBr): 3285, 3066, 2930, 1650, $1594 \mathrm{~cm}^{-1} ;{ }^{1} \mathrm{H} \mathrm{NMR}(600 \mathrm{MHz}$, $\mathrm{CDCl}_{3}$ ): $\delta 7.45$ (brs, 1H), $7.80(\mathrm{~s}, 1 \mathrm{H}), 7.02$ (dd, J 11.0, $\left.9.0 \mathrm{~Hz}, 1 \mathrm{H}\right), 6.55(\mathrm{~s}, 1 \mathrm{H}), 6.10(\mathrm{~d}, J 10.0 \mathrm{~Hz}, 1 \mathrm{H}), 5.61(\mathrm{~m}$, $2 \mathrm{H}), 4.61(\mathrm{~d}, J 5.0 \mathrm{~Hz}, 2 \mathrm{H}), 2.16(\mathrm{~m}, 2 \mathrm{H}), 2.20(\mathrm{~m}, 2 \mathrm{H}), 1.55(\mathrm{~m}, 4 \mathrm{H}) ;{ }^{13} \mathrm{C} \mathrm{NMR}\left(125 \mathrm{MHz}, \mathrm{CDCl}_{3}\right): \delta 172.9,156.3$, 
136.1, 135.9, 132.2, 128.7, 124.1, 119.4, 113.5, 112.0, 110.7, 70.1, 40.9, 28.5, 26.5, 21.1; MS(EI) m/z: 415 (M+, 45), 317 (100), 238 (70); $\mathrm{HRMS}(\mathrm{FAB})$ calcd for $\mathrm{C}_{16} \mathrm{H}_{17} \mathrm{Br}_{2} \mathrm{NO}_{2}(\mathrm{M}+\mathrm{H})^{+} 413.9626$. Found 413.9623.

\section{Acknowledgements}

We gratefully acknowledge support for this project by COSNET grant 421.03-P (2003) and CONACYT (F264E9207).

\section{References}

1. Tschesche, R.; Kaussmann, E. U. The Alkaloids: Manske, R. H. F., Ed.; Academic Press: New York; 1975; pp 164-205.

http://dx.doi.org/10.1016/S1876-0813(08)60276-6

2. Schmidt, U.; Liberknecht, A.; Haslinger, E. The Alkaloids: Brossi, A., Ed.; Academic Press: New York 1985; pp 299-326.

http://dx.doi.org/10.1016/S0099-9598(08)60197-2

3. Tan, N-H.; Zhou, J. Chem. Rev. 2006, 106, 840. http://dx.doi.org/10.1021/cr040699h

4. Joullie, M. M.; Richard, D. J. Chem. Commun. 2004, 2011.

http://dx.doi.org/10.1039/B400334A

5. Han, J.; Ji, C. J.; He, W.-J.; Shen, Y.; Leng, Y.; Xu, W.-Y.; Fan, G.-Z.; Kong, L.-D.; Tan, N.-H. J. Nat. Prod. 2011, 74, 2571. http://dx.doi.org/10.1021/np200755t

6. Kaleen, W. A.; Nisar, M.; Qayum, M.; Zia-Ul-Haq, M.; Adhikari, A.; De Feo, V. J. Mol. Sci. 2012, $13,11520$. http://dx.doi.org/10.3390/ijms130911520

7. Schmidt, U.; Lieberknecht, A.; Bökens, H.; Griesser, H. Angew. Chem. Int. Ed. 1981, 20, 1026. http://dx.doi.org/10.1002/anie.198110261

8. Laïb, T.; Zhu, J. Tetrahedron Lett. 1998, 39, 283.

http://dx.doi.org/10.1016/S0040-4039(97)10513-5

9. Lagarias, J. C.; Houghten, R. A.; Rapoport, H. J. Am. Chem. Soc. 1978, 100, 8202.

http://dx.doi.org/10.1021/ja00494a030

10. Gournelis, D. C.; Laskaris, G. G.; Verpoorte, R. Nat. Prod. Rep. 1997, 75. http://dx.doi.org/10.1039/NP9971400075

11. Zhao, S.; Juang, B.; Peng, W.; He, W.; Xu, H.; Ji, C.; Han, J.; Zhen, Y.; Song, W.; Tan, N. Chin. J. Chem. 2012, $30,1213$.

http://dx.doi.org/10.1002/cjoc.201200508

12. Goyal, M.; Prakash, B.; Sasmal, D. Spatula DD 2012, 2, 107.

http://dx.doi.org/10.5455/spatula.20120422080614

13. Zhao, S.; Kuang, B.; Peng, W.; He, W.; Xu, H.; Ji, C.; Han, J.; Zhen, Y.; Song, W.; Tan, N. Chin. J. Chem. 2012, 30, 1213.

http://dx.doi.org/10.1002/cjoc.201200508 
14. Aguirre, G.; Somanathan, R.; Hellberg, L. H. J. Fluorine Chem. 1998, 90, 5.

http://dx.doi.org/10.1016/S0022-1139(98)00143-2

15. Somanathan, R.; Rivero, I. A.; Aguirre, G.; Ramirez, M.; Hellberg, L. H.; Bakir, F. Synth. Commun. 1996, 26, 1023.

http://dx.doi.org/10.1080/00397919608003707

16. Obrecht, J.; Hellberg, L. H.; Somanathan, R. Chem. Commun. 1987, 1219.

http://dx.doi.org/10.1039/C39870001219

17. Osuna-Ramírez, M.; Aguirre, G.; Somanathan, R.; Molins, E. Tetrahedron: Asymmetry 2002, $13,2261$. http://dx.doi.org/10.1016/S0957-4166(02)00586-4

18. Osuna-Ramírez, M.; Narvaez-Garayzar, O. F.; Somanathan, R.; Aguirre, G. J. Mex. Chem. Soc. 2011, 55, 57.

19. Kirkuk, P.; Moore, R. Tetrahedron Lett. 1983, 24, 2087.

http://dx.doi.org/10.1016/S0040-4039(00)81851-1

20. Anderson, R. J. Tetrahedron Lett. 1978, 19, 2541.

http://dx.doi.org/10.1016/S0040-4039(01)94821-X

21. Anderson, R. J.; Stonard, R. J. Can. J. Chem. 1979,57, 2325.

http://dx.doi.org/10.1139/v79-372

22. Bruening, R. C.; Oltz, E. M.; Furakawa, J.; Nakanishi, K.; Kustin, K. J. Nat. Prod. 1986, 49, 193. http://dx.doi.org/10.1021/np50044a001

23. Oltz, E. M.; Bruening, R. C.; Smith, M. J.; Kustin, K.; Nakanishi, K. J. Am. Chem. Soc. 1988, 110, 6162. http://dx.doi.org/10.1021/ja00226a035

24. Azumi, K.; Yokisawa, H.; Ishii, S. Biochemistry 1990, 29, 159.

http://dx.doi.org/10.1021/bi00453a021

25. Ohkuma, K.; Anzai, K.; Suzuki, S. J. Antibiot. 1962, 15, 115.

26. Umezawa, H.; Imoto, M.; Sawa, T.; Isshiki, K.; Matsuda, M.; Uchidda, T.; Linuma, H.; Hamada, M.; Takeuchi, T. J. Antibiot. 1986, 39, 170.

http://dx.doi.org/10.7164/antibiotics.39.170

27. Kagamizono, T.; Sakai, N.; Arai, K.; Kobinata, K.; Osada, H. Tetrahedon Lett. 1997, 38, 1223.

http://dx.doi.org/10.1016/S0040-4039(97)00022-1

28. Chatterjee, A.; Chakrabarty, M.; Kundu, A. B. Austr. J. Chem. 1975, 28, 457.

http://dx.doi.org/10.1071/CH9750457

29. Govidachari, T.; Premila, M. Phytochemistry 1983, 22, 755.

http://dx.doi.org/10.1016/S0031-9422(00)86977-0

30. Burke, B. A.; Philip, S. Heterocycles 1985, 23, 257.

http://dx.doi.org/10.3987/R-1985-02-0257

31. Burke, B. A.; Perkins, H. Tetrahedron Lett. 1978, 19, 2723.

http://dx.doi.org/10.1016/S0040-4039(01)91587-4

32. Galanis, D. L.; McKee, T. C.; Pannell, L. K.; Cardellina, J. H.; Boyd, M. R. J. Org. Chem. 1997, 62, 8968. http://dx.doi.org/10.1021/jo9716237

33. Kim, J. W.; Shin-Ya, K.; Furihata, K.; Hyakawa, Y.; Seto, H. J. Org. Chem. 1999, 64, 153-155. http://dx.doi.org/10.1021/jo9814997

34. Erickson, K. L.; Beutler, J. A.; Cardellina II, J. H.; Boyd, M. R. J. Org. Chem. 1997, 62, 8188. http://dx.doi.org/10.1021/jo971556g

35. Erickson, K. L.; Beutler, J. A.; Cardellina II, J. H.; Boyd, M. R. J. Org. Chem. 2001, 66, 1532. http://dx.doi.org/10.1021/jo004040x 
36. Jensen, R.; Kunze, B.; Reichenbach, H.; Höfle, G. Eur. J. Org. Chem. 2000, 913.

http://dx.doi.org/10.1002/(SICI)1099-0690(200003)2000:6<913::AID-EJOC913>3.0.CO;2-L

37. McKee, T. C.; Galinis, D. L.; Pannell, L. K.; Cardellina II, J. H.; Laakso, J.; Ireland, C. M.; Murray, L.; Capon, R. J.; Boyd, M. R. J. Org. Chem. 1998, 63, 7805.

http://dx.doi.org/10.1021/jo980939r

38. Suzumura, K.-I.; Takahashi, I.; Matsumoto, H.; Nagai, K.; Setiawan, B.; Rantiatmodjo, R. M.; Suzuki, K.-I.; Nagano, N. Tetrahedron Lett. 1997, 38, 7573.

http://dx.doi.org/10.1016/S0040-4039(97)01785-1

39. Xie, X.-S.; Padron, D.; Liao, X.; Wang, J.; Roth, M. G.; de Brabander, J. K. J. Biol. Chem. 2004, $279,19755$. http://dx.doi.org/10.1074/ibc.M313796200

40. Wu, Y.; Esser, L.; De Brabander, J. K. Angew. Chem. Int. Ed. 2000, 39, 4308.

http://dx.doi.org/10.1002/1521-3773(20001201)39:23<4308::AID-ANIE4308>3.0.CO;2-4

41. Wu, Y.; Seguil, O. R.; De Brabander, J. K. Org. Lett. 2000, 2, 4241.

http://dx.doi.org/10.1021/ol0068086

42. Wu, Y.; Liao, X.; Wang, R.; Xie, X.; De Brabander, J. K. J. Am. Chem. Soc. 2002, 124, 3245.

http://dx.doi.org/10.1021/ja0177713

43. Shih, C. ; Teicher, B. A. Current Pharm. Design 2001, 7, 1259.

https://doi.org/10.2174/1381612013397474

44. Stachel, S. J.; Chapell, M. D.; Lee, C. B.; Danishefsky, S. J.; Chou, T.-C.; He, L.; Horwitz, S. B. Org. Lett. 2000, 2, 1637.

http://dx.doi.org/10.1021/ol005932m

45. Dimopoulos, M. A.; Mitsiades, C. S.; Anderson, K. C.; Richardson, P. G. Cl. Lymph. Myelom. Leuk. 2011, $11,17$.

46. Kimura, T.; Iwatsuki, M.; Asami, Y.; Ishiyama, A.; Hokari, R.; Otoguro, K.; Matsumoto, A.; Sato, N.; Shiomi, K.; Takahashi, Y.; Omura, S.; Nakashima, T. J. Antibiot. 2016, 69, 818.

http://dx.doi.org/10.1038/ja.2016.28

47. Heffner, R. J.; Jiang, J.; Joullie, M. M. J. Am. Chem. Soc. 1992, 114, 10181.

http://dx.doi.org/10.1021/ja00052a015

48. Jacobsen, O.; Klaveness, J.; Rongved, P. Molecules 2010, 15, 6638.

http://dx.doi.org/10.3390/molecules15096638

49. Yu, X.; Sun, D. Molecules 2013, 18, 6230.

http://dx.doi.org/10.3390/molecules18066230

50. Liu, X.; Sternberg, E.; Dolphin, D. J. Org. Chem. 2008, 73, 6542.

http://dx.doi.org/10.1021/jo8007989

51. Mang, S. L.; Grubbs, R. H. Chem. Sci. 2015, 6, 4561.

http://dx.doi.org/10.1039/c5sc01507c

52. Gradillas, A.; Pérez-Castells, J. Angew. Chem. Int. Ed. 2006, 106, 6086.

http://dx.doi.org/10.1002/anie.200600641

53. Scheiper B.; Glorius F.; Leitner A.; Fürstner A. PNAS 2004, 11960.

http://dx.doi.org/10.1073/pnas.0401322101

54. Wang, C.; Yu, M.; Kyle, A. F.; Jakubec, P.; Dixon, D. J.; Schrock R. R.; Hoveyda A. H. Chem. Eur. J. 2013, 19, 2726.

http://dx.doi.org/10.1002/chem.201204045 
55. Wang Y.; Jimenez M.; Sheehan P.; Zhong C.; Hung A. W.; Tam C. P.; Young D. W. Org. Lett. 2013, 15, 1218.

http://dx.doi.org/10.1021/ol400134d

56. Majumdar K. C.; Rahaman H.; Roy B. Curr. Org. Chem. 2007, 11, 1339.

http://dx.doi.org/10.2174/138527207782023166

57. Toumi, M.; Couty, F.; Evano, G. J. Org. Chem. 2008, 73, 1270.

http://dx.doi.org/10.1021/jo702092x

58. Prabhakaran, E. N.; Rao, N.; Boruah, A.; Iqbal, J. J. Org. Chem. 2002, 67, 8247.

http://dx.doi.org/10.1021/jo0200320

59. Banerji, B.; Mallesham, B.; Kumar, S. K.; Kunwar, A. C.; Iqbal, J. Tetrahedron Lett. 2002, 43, 6479. http://dx.doi.org/10.1016/s0040-4039(02)01239-x

60. Harris, P. W. R.; Brimble, M. A.; Gluckman, P. D. Org. Lett. 2003, 11, 1847.

http://dx.doi.org/10.1021/ol034370e 